\title{
Effect of 6Minute Walk Test on Cardiac Parameters in Pediatric Age Group
}

\author{
Rajak P.S. ${ }^{1}$, Malwade M. ${ }^{2}$,Khatri S.M. ${ }^{3}$, Kathariya N. ${ }^{4}$ \\ ${ }^{I}$ Post Graduate student of Department Of Cardio- Respiratory Physiotherapy, College Of Physiotherapy, PIMS, \\ Loni, Ahmednagar. \\ ${ }^{2}$ Asso.Professor of Department Of Paediatric Physiotherapy, College Of Physiotherapy, PIMS, Loni, \\ Ahmednagar \\ ${ }^{3}$ Principal,College Of Physiotherapy, PIMS, Loni, Ahmednagar. \\ ${ }^{4}$ Post- Graduate student, Department Of Anaesthesiology, Rural Medical College, PIMS, Loni, Ahmednagar.
}

\begin{abstract}
Objective: To study the effect of 6 Minute Walk Test on cardiac parameters in paediatric age group and put forth values post $6 M W T$ for reference.

Materials and Methods: This study was conducted in 31 healthy normal children studying in a local school. They were selected independent of gender and after screening. The parameters emphasized were Respiratory rate, Pulse rate, $V O_{2 M a x}$ and blood pressure. During screening a routine BMI, Past medical history (school records and parental interview), Auscultation, Pulse rate, Respiratory rate, Blood pressure were noted. . Result: There was a mild increase in the Respiratory rate, Pulse rate, Blood pressure post test, while higher values were noted in all the parameters in girls. The $\mathrm{VO}_{2 . \text { Max }}$ values calculated also showed a higher value for girls in comparison to boys.

Conclusion: 6 Minute Walk Test is safe in healthy normal children, it provides a mild rise in cardiac parameters but within the available normal range. It also states higher values in girls as compared to boys.

Key Words: 6MWT, Cardiac parameters, Paediatric Age Group, Reference Values
\end{abstract}

\section{Introduction:}

The heart as we all know is the whole and soul of human body. Its position and circulation are of utmost importance. Any disturbance in it leads to a series of life threatening events. Thus it is essential to study its response to various physical activities from childhood to ageing.

The best method to study the heart, is by exercise testing which provides information on exercise capacity and facilitates assessment of patho-physiologic characteristics, effectiveness of medication and risk of potential disease. The composite assessment of respiratory, cardiac and metabolic systems depends on an individual's response to exercise . Since, most ADLs are performed at submaximal level of exertion, using submaximal functional tests could provide a more realistic simulation to one's physical capability ${ }^{1}$

The components for choosing such a test method depend on its ease, duration, cost effectiveness , accuracy of results and its implications in real life. Based on these few points BALKE developed a simple test to examine functional capacity by measuring the distance walked during a specified period of time. The original test was conducted for a period of 12 minutes, to assess an individual's physical fitness, which later in time got modified and is used as 6 min walk test due to its less demanding nature and ability to provide equally good comparable data. ${ }^{2}$

A recent review of functional test also concluded that 6MWT is easy to perform with better acceptibility and provides better reflection of activities of daily living than other walk tests.

Here, the 6mins walk test is chosen as an outcome measure to determine the endurance performance of normal healthy children . The test is valid for children and correlates with the result of treadmill testing $(\mathrm{r}=0.39)$, the shuttle run test $(r=0.88)$ and metabolic parameters such as lactate $(r=0.92)$ ( Beck and Bo's: 1995 , Bo's 2001)

The distance covered in $6 \mathrm{mins}$ has been shown to accurately predict the mortality and morbidity from cardio pulmonary disease .( Jun T Au, Hung K So 2006). However, previous studies conducted indicate and promote the use of test in groups of diseased patients with physical dysfunction and did not include normal healthy subjects. The lack of 6MWT reference values from normal healthy children raises a bar in sanctioning the clinical utilization of this test in paediatric age group.( AMJ RespiCrit Care Vol. 176)

1)Study design : Observational

\section{Materials And Methods:}

2) Sample studied : 31

3)Source of data : Ahilyabai holkar school,Loni

4)Sample technique : Simple random sampling 
5) Inclusion criteria :

$>$ Children with Age group 5-6yrs

$>$ Both Male - Female

6)Exclusion critertia :

Children with:

$>$ Any past-medical history

$>$ Any congenital diseaselmusculoskeletal deformity

$>$ Any neurological deformity

$>$ Any visual/ hearing deficit

7)Materials :

$>$ Measuring tape

$>$ Calibrated Weighing machine

$>$ Timer

$>$ Calibrated BP Apparatus

8) Test used : 6Min. Walk test

9) Parameters calculated :

$>$ Pulse rate

$>$ Respiratory rate

$>$ Blood pressure

$>\mathrm{VO}_{2}$ Max.

10) $\mathrm{VO}_{2 \text { Max }}$ formula

$>132.853-(0.0769 \times$ Wt. $)-(0.3877$ x Age $)+(6.315$ x Gender $)-(3.2649$ x Time $)-(0.1565 \times$ Heart rate )

\section{Collection of Sample:}

The written consent of the concerned school authority and parents was obtained prior to any initiation of the test. The procedure was duly explained to the staff and parents. First, the basal parameters of height, weight, BMI, pulse rate. Respiratory rate, blood pressure were noted. The child was explained the way to perform the test .The timer was then set to six minutes and a therapist accompanied the child. The child was instructed to report any discomfort during the test to the accompanying physiotherapist. At the end of 6mins. , the same baseline parameters viz., pulse rate , respiratory rate and blood pressure were noted and $\mathrm{VO}_{2}$ max. was calculated. After completion of the test and recording of the parameters, the collected data was tabulated and presented as statistical analysis

\section{Observation and Results:}

Table1:

Puts forth the pre and post avg. values of pulse rate and respiratory rate

\begin{tabular}{|l|l|l|}
\hline Parameter & Pre & Post \\
\hline Pulse Rate & 99 & 108 \\
\hline Respiratory Rate & 23 & 27 \\
\hline
\end{tabular}

Table2:

Average blood pressure value, pre and post 6MWT .

\begin{tabular}{|l|l|l|}
\hline PARAMETERS & PRE & POST \\
\hline SYSTOLE & 123 & 135 \\
\hline DIASTOLE & 83 & 91 \\
\hline
\end{tabular}

Table 3:

Average values cardiac parameters distributed according to sex

\begin{tabular}{|l|l|l|l|l|}
\hline PARAMETERS & PR.(Pre) & PR.(Post) & RR.(Pre) & RR.(Post) \\
\hline GIRLS & 107 & 117 & 26 & 29 \\
\hline BOYS & 91 & 99 & 21 & 23 \\
\hline
\end{tabular}

Table 4 : 
Average values of blood pressure values before and after the test according to sex.

\begin{tabular}{|l|l|l|}
\hline PARAMETERS & BP Systole & BP Diastole \\
\hline GIRLS & 147 & 97 \\
\hline BOYS & 125 & 85 \\
\hline
\end{tabular}

Table 5:

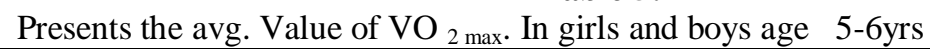

\begin{tabular}{|l|l|}
\hline PARAMETER & $\mathrm{VO}_{2 \text { Max. }}$ \\
\hline GIRLS & 88 \\
\hline BOYS & 79 \\
\hline
\end{tabular}

$>1$ shows the average values of pulse rate and respiratory rate before and after the 6MWT. The value obtained is PR. (Pre $=99 \mathrm{bpm}$ Post $=108 \mathrm{bpm})$ and RR. (Pre $=23 \mathrm{cpm}$ Post $=27 \mathrm{cpm})$. The graph shows a mild rise in these parameters .

$>2$ represents the avg. Blood pressure values recorded before and after the 6MWT, it also shows a mild rise. BP $($ Pre $=123 / 83 \mathrm{mmhg}$ Post $=135 / 91 \mathrm{mmhg})$

$>3$ presents the sex wise variation of pulse rate and respiratory rate in children aged 5-6yrs. It also records a high reading for girls in comparison to boys. PR Pre (Girls = 107bpm; Boys $=91 \mathrm{bpm})$ : PR Post $($ Girls $=$ $117 \mathrm{bpm}$; Boys $=99 \mathrm{bpm})$, whereas the respiratory rate is RR Pre ( Girls= 26cpm; Boys $=21 \mathrm{cpm}): \mathrm{RR}$ Post $($ Girls $=29 \mathrm{cpm} ;$ Boys $=23 \mathrm{cpm})$

$>4$ summarises the avg. Blood pressure values before and after the $6 \mathrm{MWT}$ as per the sex wise distribution. It also shows relatively high value for girls than boys. BP Pre ( Girls=125/118mmhg ; Boys= 114/79mmhg) BP Post ( Girls=146/97mmhg ; Boys=125/85mmhg).

$>5$ shows the calculated value of $\mathrm{VO}_{2}$ Max as per the formula with sex wise distribution. Here also higher values are obtained for girls in comparison to boys. VO2 Max. ( Girls $=88$; Boys $=79$ )

\section{Discussion:}

According to the study conducted, the average values for girls and boys aged 5-6yrs have been found. These values denote a mild rise after the participants underwent 6MWT a sub-maximal walk test. The reason for this mild rise is the physiologic response of the human body.

The parameters calculated i.e. the pulse rate, respiratory rate and the blood pressure are vital signs showing an image of body's internal condition.

The vitals also show gender differences. The values obtained for pulse rate of total population under study are: before the test $(99 \mathrm{bpm})$ and after the test $(110 \mathrm{bpm})$; the respiratory rate is pre $(23 \mathrm{cpm})$ and post $(27 \mathrm{cpm})$; blood pressure pre $(113 / 78 \mathrm{mmHg})$ and post $(120 / 80 \mathrm{~mm} \mathrm{Hg})$. Thus, we note there is an increase in every parameter after the 6MWT.

This is due to an increase in demand of oxygen by the muscles due to exertion. The physical exertion causes the muscles of the body to contract and burns the oxygen in blood leading to an increase in its demand. This increase in demand is fulfilled by the heart via an increase in its contraction and more pumping of blood to the contracting muscle. As this process advances with increasing physical exertion the workload on vital organs increases. Thus this increased work causes a rise in the basal parameters .

The reason for females having higher parameters than males can be due to more physical exertion along with school and playing activities, which is fairly common in Indian scenario.

\section{Acknowledgement:}

The authors are thankful to the Rajak family, Principal of Ahilyabai Holkar school, Rashmi Bhaval, Dr. Neesha Shinde (HOD Cardio - Respiratory Sciences),

\section{References:}

[1]. Nixon PA,Joswiak ML, Fricker FJ.A six minute walk test for assessing exercise tolerance in severely ill children .J Pediatr.1996;129:362-366

[2]. Solway S,Brooks D,Lacasse Y,Thomas S.A qualitative systematic overview of the measurement properties offunctional walk tests used in the cardio respiratory domain.Chest.2001;119:256-270

[3]. Li AM,Yin J,Au JT,So HK,Tsang T,Wong E,et al.Standard reference for the six-minute-walk test in healthy children aged 7 to 16 years.Am J Respir Crit Care Med.2007;176:174-180

[4]. Geiger R, Strasak A, Treml B, Gasser K, Kleissner A, Fischer V, et al. Six minute walk test in children and adolscents. JPediatr.2007:150;395-399.

[5]. Gulmans VA, van Veldhoven NH, de meer K, Helder PJ. The six minute walking distance in children with cystic fibrosis: reliability and validity. Pediatr Pulmonal.1996;22:85-90. 
[6]. Lersson UE, Reynisdottir S. The six minute walk test in outpatients with obesity: reproducibility and known group vaidity.Physiother Res Int. 2008;13:84-93

[7]. Li AM, Yin J, Yu CCW, Tsang T, So HK, Wong E,et al. The six minute walk test in healthy children: reliability and validity. Eur Respir J.2005;25:1057-1060.

[8]. Calders P, Deforche B, Verschelde S, Bouckaert J, et al. Predictors of 6minute walk test and 12 minute walk/run test in obese children and adolscents. Eur J Pediatr.2008;167:563-568

[9]. Morinder G, Mattsson, Sollander C, Evers Larsson U, Marcus C. Six minute walk test in obese children and adolscents: eproducibility and validity. Physioth Re Int. 2009;14:91-104

[10]. American Thoracic Society, ATS Statement guidelines for the six minute walk test. Am J Respir Crit Care Med.2002;166:111-117.

[11]. Deurenberg P,Weststrate JA, Seidell JC. Body mass index as a measure of body fatness: age and sex specific prediction formula s. Br J Nutr.1991;65:105-114. 